\title{
Prevalência de defeitos de fechamento de tubo neural no Vale do Paraíba, São Paulo
}

\author{
Prevalence of neural tube defects in Vale do Paraíba, São Paulo, Brazil
}

\author{
Luiz Fernando C. Nascimento ${ }^{1}$
}

\section{RESUMO}

Objetivo: Estimar a prevalência de defeitos de fechamento do tubo neural no Vale do Paraíba paulista e identificar possíveis fatores maternos e neonatais associados a tais defeitos.

Métodos: Realizou-se um estudo transversal com dados secundários obtidos na Secretaria Estadual da Saúde referentes aos nascimentos ocorridos em 2004 no Vale do Paraíba paulista, que compreende 35 municípios e conta com população de 2 milhões de habitantes. Anencefalia, encefalocele e espina bífida (mielocele e mielomeningocele) foram considerados defeitos de fechamento do tubo neural. As variáveis maternas foram: idade, escolaridade, cor da pele, número de consultas no pré-natal, número de filhos vivos e relato de óbito fetal prévio. As variáveis relativas ao recém-nascido foram: peso, idade gestacional e escore de Apgar. Realizou-se comparação das médias por meio do teste $t$ de Student e obtiveram-se os valores das razões de chance com intervalos de confiança de $95 \%$.

Resultados: Foram analisados 33.653 nascidos vivos. Trinta e oito recém-nascidos com o defeito foram encontrados (1,13/1.000 nascidos vivos), sendo 23 casos de espina bífida. Houve associação com baixo peso ao nascimento, prematuridade e menores escores de Apgar de cinco minutos.

Conclusões: A prevalência desta anomalia foi inferior à de outros estudos nacionais e sua presença esteve associada ao baixo peso, à prematuridade e à baixa vitalidade ao nascer.

Palavras-chave: defeitos do tubo neural; anormalidades congênitas; declaração de nascido vivo; nascidos vivos.

\section{ABSTRACT}

Objective: To estimate the prevalence of neural tube defects in Vale do Paraíba, São Paulo, Brazil, and to identify possible maternal and neonatal variables associated with these defects.

Methods: This cross-sectional study used secondary records of the Health Department of São Paulo State related live births during 2004 in Vale do Paraíba, São Paulo, Brazil. This region has 35 cities and 2 million inhabitants. Anencephaly, encephalocele and spina bifida (myelocele and myelomeningocele) were considered as neural tube defects. The following maternal variables were analyzed: age, educational level, race, number of born alive and stillborn infants and prenatal visits. Neonatal variables were: birth weight, gestational age and Apgar score. Numerical variables were compared by Student $t$ test, and Odds Ratio values were obtained with the $95 \%$ confidence interval.

Results: The analysis was performed based on 33,653 records of born alive infants. Twenty-three infants with spina bifida were identified, with an estimated prevalence of 1.13 cases for each 1,000 live births. The presence of neural tube defects was associated to low birth weight, prematurity and low Apgar score.

Conclusions: The estimated prevalence of neural tube defects in this region of São Paulo was lower than others reported in previous Brazilian studies. These defects were associated with low birth weight, prematurity and respiratory depression at birth.

Key-words: neural tube defects; congenital abnormalities; birth certificate; live birth.
Instituição: Universidade de Taubaté (Unitau), Taubaté, SP, Brasil 1Professor-assistente do Departamento de Medicina da Unitau, doutor em Saúde Pública pela Universidade de São Paulo, São Paulo, SP, Brasil
Endereço para correspondência:

Luiz Fernando C. Nascimento

Avenida Tiradentes, 500 - Bom Conselho

CEP 12030-180 - Taubaté/SP

E-mail: Ifcn@unitau.br

Recebido em: 23/4/2008

Aprovado em: 2/7/2008 


\section{Introdução}

Os defeitos de fechamento do tubo neural (DTN) são malformações congênitas que ocorrem com relativa freqüência e decorrem de uma falha no adequado fechamento do tubo neural embrionário, por volta da quarta semana de embriogênse ${ }^{(1,2)}$. Tais defeitos incluem principalmente anencefalia, meningocele, mielomeningocele e encefalocele. Etiologicamente envolvem fatores genéticos e ambientais e, geralmente, são multifatoriais ${ }^{(1,3)}$.

Sua prevalência é cerca de um caso em cada 1.000 nascidos $\operatorname{vivos}^{(4)}$, mas pode atingir 4,73 por 1.000 nascidos vivos ${ }^{(5)}$, como encontrado em Minas Gerais, ou cinco casos por 1.000 nascidos vivos, como verificado em Recife ${ }^{(6)}$, Pernambuco. Valores mais baixos de prevalência foram encontrados em São José dos Campos, São Paulo, com 1,2 caso por 1.000 nascidos vivos $^{(7)}$. Em investigação do Estudo Colaborativo LatinoAmericano de Malformações Congênitas (ECLAMC), entre 1967 e 1995 no Chile, foram analisados cerca de 4 milhões de nascidos vivos, observando-se defeitos de fechamento do tubo neural em 1,5 por 1.000 nascidos vivos ${ }^{(8)}$.

Os defeitos de fechamento de tubo neural têm papel relevante na morbimortalidade infantil. Na maioria dos casos de anencefalia ocorre o óbito fetal ou as crianças morrem logo após o nascimento. Os pacientes portadores de mielomeningocele ou meningocele podem apresentar comprometimento grave de sua capacidade motora como paralisia dos membros inferiores, hidrocefalia e disfunções vesical e intestinal.

A prevenção dos defeitos de fechamento de tubo neural pode ser feita por meio da ingestão de $0,4 \mathrm{mg} / \mathrm{dia}$ de ácido fólico por mulheres sem antecedentes de concepto com a malformação e de $4 \mathrm{mg} /$ dia naquelas com antecedentes ${ }^{(9)}$. $\mathrm{O}$ ácido fólico deve ser ingerido no período periconcepcional (pelo menos dois meses antes da concepção) e no primeiro trimestre da gravidez. Diante da evidência do papel protetor do ácido fólico, o Ministério da Saúde brasileiro regulamentou a fortificação de farinhas de trigo e de milho com ferro e ácido fólico ${ }^{(10)}$. No entanto, ainda há desconhecimento sobre o mecanismo que regula o efeito protetor do ácido fólico em relação aos defeitos de tubo neural.

Com a introdução do campo 34 na Declaração de Nascido Vivo do Ministério da Saúde, indicador de presença ou não de malformações congênitas e/ou anomalia cromossômica, tornou-se mais fácil estimar a prevalência de defeitos no tubo neural nos nascimentos no Brasil, mesmo não haven- do possibilidade de identificação de defeitos múltiplos ou associados.

O objetivo deste trabalho, realizado em 2004, foi estimar a prevalência de defeitos no tubo neural na cidade do Vale do Paraíba e identificar as possíveis associações com fatores maternos e do recém-nascido, haja vista a inexistência desse tipo de estudo na região.

\section{Métodos}

Trata-se de estudo transversal com dados secundários referentes aos nascidos vivos no ano de 2004 na região do Vale do Paraíba obtidos a partir do portal da Secretaria Estadual de Saúde de São Paulo. A cidade em questão contém 35 municípios e cerca de 2 milhões de habitantes.

A variável dependente foi presença de defeito no fechamento do tubo neural, identificado pelos códigos Q00 (anencefalia), Q01 (encefalocele) e Q05 (espina bífida, que inclui meningocele e mielomeningocele) da Classificação Internacional de Doenças, $10^{a}$ versão (CID-10).

As variáveis independentes foram divididas em maternas e do recém-nascido. As maternas foram: idade e escolaridade, número de filhos vivos em gestações anteriores, presença de óbito fetal no passado gestacional e obstétrico, número de consultas realizadas no pré-natal e cor da pele. As variáveis coletadas do recém-nascido foram: peso ao nascer, duração da gestação e escore de Apgar de cinco minutos. Todas estas informações constam na Declaração de Nascido Vivo.

A idade materna foi estimada como variável quantitativa segundo a presença ou não de defeito no fechamento do tubo neural, sendo que o grupo que não apresentava tais defeitos poderia ser portador de outras malformações. Em seguida, a idade foi categorizada em três grupos: mães de até 19 anos, de 20 a 34 anos e com idade igual ou superior a 35 anos. O número de filhos foi classificado como: nenhum, um filho e dois ou mais filhos. O relato de óbito fetal foi considerado presente ou ausente. A escolaridade materna foi dividida em: até quatro anos (parte do ensino fundamental) e cinco ou mais anos de escolaridade (término do ensino fundamental, ensino médio e superior). O número de consultas de pré-natal foi dividido em: até seis consultas e sete ou mais consultas. As mães foram categorizadas em brancas e não-brancas, segundo a cor da pele. 
As variáveis analisadas com relação ao recém-nascido foram: peso ao nascer (em gramas), analisado como variável quantitativa e depois categorizado em baixo peso $(<2.500 \mathrm{~g})$ e peso normal ( $\geq 2.500 \mathrm{~g})$. Quanto à duração da gestação, foi considerado pré-termo o nascimento com menos de 37 semanas de gestação e a termo aquele com 37 semanas ou mais de gestação. O escore de Apgar de cinco minutos foi analisado como variável quantitativa e, posteriormente, categorizada em: baixa vitalidade $(<6)$ e boa vitalidade $(\geq 7)$.

As variáveis quantitativas foram estimadas e as médias foram comparadas pelo teste $t$ de Student, segundo a presença ou não de defeito no fechamento do tubo neural. As variáveis categóricas foram analisadas pelo teste do qui-quadrado ou teste exato de Fisher, quando indicado. Por fim, as variáveis ordinais foram analisadas pelo teste do qui-quadrado de tendência linear.

Como esses são eventos raros, os riscos foram estimados pelos valores da razão de chance (Odds Ratio-OR). Foram estimados os intervalos de confiança de $95 \%$ para cada defeito do tubo neural, utilizando-se a distribuição de Poisson. Os totais de cada variável podem diferir entre si, pois não foram excluídos os dados ignorados e não preenchidos.

Os resultados, apresentados em tabelas, referem-se aos dados obtidos a partir das Declarações de Nascido Vivo do ano de 2004 de 35 municípios do Vale do Paraíba paulista. Eventualmente, o total de defeitos no fechamento do tubo neural, de acordo com determinada variável independente, pode ser diferente do total encontrado devido à ausência de informação relativa a tal variável independente.

Os dados foram analisados com o programa Epi-Info 6.04. O qui-quadrado utilizado foi o de Mantel-Hantzel. Considerou-se $p<0,05$ significante.

\section{Resultados}

Foram estudadas 33.653 Declarações de Nascido Vivo relativas aos partos ocorridos no ano de 2004 na região.

Tabela 1 - Distribuição dos 45 defeitos de fechamento do tubo neural, com os respectivos intervalos de confiança de $95 \%$, Vale do Paraíba, São Paulo, 2004

\begin{tabular}{lcc}
\hline & $\mathbf{n ~ ( \% ) ^ { \# }}$ & $\mathbf{I C 9 5 \%}$ \\
\hline Anencefalia & $14(36,8)$ & $8-21$ \\
Encefalocele & $1(2,6)$ & $0-3$ \\
Espina bífida & $23(60,6)$ & $14-31$ \\
\hline
\end{tabular}

\#porcentagens em relação ao total de defeitos de fechamento do tubo neural.
Identificaram-se 274 anomalias congênitas $(0,81 \%$ dos partos), sendo que 38 casos eram de defeito no fechamento do tubo neural, representando $13,8 \%$ de todas as anomalias congênitas identificadas. Houve prevalência de 1,34 defeito de fechamento de tubo neural a cada 1.000 nascidos vivos. O não-preenchimento do campo 34 da DNV ocorreu em 27 casos $(0,08 \%)$.

Os defeitos de fechamento de tubo neural foram mais prevalentes no sexo feminino do que no masculino $(70,4$ versus 29,6\%), tendo ocorrido em um recém-nascido com sexo indeterminado. Foram feitos 30 cesáreas e oito partos normais para bebês portadores da malformação.

A idade média das mães dos pacientes com defeito no fechamento de tubo neural foi $25,0 \pm 6,8$ anos e das mães de bebês sem a anomalia foi $25,8 \pm 6,3$ anos, sem diferença estatística. Quanto ao peso médio ao nascer, os portadores do defeito apresentaram $2664 \pm 886 \mathrm{~g}$ e os não-portadores tinham peso de $3143 \pm 529 \mathrm{~g}$. Esta diferença foi estatisticamente significante $(p<0,001)$. Da mesma forma, os neonatos com defeito de tubo neural apresentaram escore médio de Apgar de cinco minutos de 7,5 $\pm 2,6$; os recém-nascidos sem a malformação, de $9,3 \pm 0,8$, diferença esta também significante $(p<0,001)$.

Os defeitos de fechamento no tubo neural encontrados nessa população foram: quatorze relatos de anencefalia $(0,42$ casos $/ 1.000 \mathrm{NV})$, um caso de encefalocele $(0,03$ casos $/ 1.000 \mathrm{NV})$ e 23 casos de espina bífida $(0,68$ casos/1.000 NV). Esses valores e respectivas porcentagens em relação ao total de defeitos de fechamento de tubo neural juntamente com os intervalos de confiança de $95 \%$ obtidos através da distribuição de Poisson são apresentados na Tabela 1.

Quanto à ligação de variáveis maternas com os defeitos de tubo neural (Tabela 2), pode-se observar que as mães mais jovens e com mais de 34 anos tiveram $45 \%$ mais risco de nascimento com a anomalia, mas estes valores não apresentaram significância estatística. O risco foi mais elevado nas mães com pouca escolaridade, menor número de consultas, com dois ou mais filhos e de raça branca. No entanto, tais valores também não apresentaram significância estatística.

No que diz respeito à associação de variáveis neonatais à anomalia (Tabela 3), foi possível encontrar tal associação com baixo peso, prematuridade e baixa vitalidade ao nascer. $\mathrm{O}$ risco de ocorrência do defeito de fechamento de tubo neural associado às variáveis acima descritas foi de 6 a 12 vezes maior. 
Tabela 2 - Distribuição dos valores das variáveis maternas segundo a presença ou não de defeitos de fechamento de tubo neural, Vale do Paraíba, São Paulo, 2004

\begin{tabular}{|c|c|c|c|c|}
\hline & DTN presente & DTN ausente & $p$ & OR (IC95\%) \\
\hline \multicolumn{5}{|l|}{ Idade materna } \\
\hline até 19 anos & 10 & 5.790 & $0,17^{\#}$ & $1,83(0,81-4,02)$ \\
\hline 20 a 34 anos & 23 & 24.336 & & 1,00 \\
\hline 35 anos e mais & 5 & 3.482 & & $1,52(0,51-4,21)$ \\
\hline \multicolumn{5}{|l|}{ Escolaridade } \\
\hline até 4 anos & 5 & 2.079 & 0,09 & $2,21(0,76-5,92)$ \\
\hline 5 anos e mais & 33 & 30.271 & & \\
\hline \multicolumn{5}{|l|}{ Consultas } \\
\hline até 6 & 4 & 1.635 & 0,11 & $2,25(0,68-6,65)$ \\
\hline 7 ou mais & 34 & 31.324 & & \\
\hline \multicolumn{5}{|l|}{ Filhos vivos } \\
\hline 0 & 16 & 10.634 & $0,36^{\#}$ & 1,00 \\
\hline 1 & 8 & 9.737 & & $1,83(0,74-4,66)$ \\
\hline 2 ou mais & 13 & 8.693 & & $1,01(0,45-2,22)$ \\
\hline \multicolumn{5}{|l|}{ Óbito fetal } \\
\hline não & 25 & 21.591 & 0,96 & $0,98(0,32-3,31)$ \\
\hline $\operatorname{sim}$ & 4 & 3.372 & & \\
\hline \multicolumn{5}{|l|}{ Cor } \\
\hline branca & 36 & 24.020 & 0,44 & $1,74(0,41-10,43)$ \\
\hline não-branca & 2 & 2.317 & & \\
\hline
\end{tabular}

\#obtido pelo qui-quadrado de tendência linear.

Tabela 3 - Distribuição dos valores das variáveis neonatais segundo a presença ou não de defeitos de fechamento do tubo neural, Vale do Paraíba, São Paulo, 2004

\begin{tabular}{lcccc}
\hline & DTN presente & DTN ausente & $\boldsymbol{p}$ & OR (IC95\%) \\
\hline Pré-termo & 11 & 2.197 & $<0,001$ & $5,80(2,70-12,20)$ \\
Termo & 27 & 31.272 & & \\
Baixo peso & 15 & 2.997 & $<0,001$ & $6,69(3,32-13,37)$ \\
Peso normal & 23 & 30.741 & & \\
Boa vitalidade & 8 & 762 & $<0,001$ & $11,38(4,80-26,02)$ \\
Baixa vitalidade & 30 & 32.520 & & \\
\hline
\end{tabular}

\section{Discussão}

Os valores encontrados nesta pesquisa sobre a prevalência de defeitos de tubo neural (1,13 casos/1.000 nascidos vivos) foram inferiores aos achados em Minas Gerais e Pernambuco ${ }^{(5,6)}$, possivelmente pelo fato desses dois estados envolverem hospitais de referência para onde são encaminhadas gestações de risco, aumentando assim a freqüência de nascimento com esta anomalia.

Os achados no Vale do Paraíba estão próximos aos encontrados no estudo de ECLAMC, realizado no Chile ${ }^{(8)}$, e pouco inferiores aos verificados por Costa et al no Rio de Janeiro (1,7 casos por 1.000 nascidos vivos) $)^{(11)}$. As diferenças notadas podem ser explicadas pela heterogeneidade da fonte de dados dos estudos.
A importância do presente relato reside na inexistência de estudos recentes sobre a prevalência de defeitos no fechamento do tubo neural tanto no Vale do Paraíba como no Estado de São Paulo. Além disso, esta investigação fornece dados referentes ao período anterior à política de enriquecimento das farinhas que servirão de base de comparação para estudos que objetivem avaliar a efetividade dessa política.

A inclusão do campo 34 na Declaração de Nascido Vivo facilita sobremaneira a obtenção de dados sobre anomalias congênitas e, no caso dos defeitos de tubo neural (facilmente identificáveis ao exame físico do recém-nascido), os dados regionais poderão ser comparados.

A prevalência de 1,13 casos por 1.000 nascidos vivos se aproxima da obtida nos Estados Unidos ${ }^{(4,12)}$ : um caso para 
1.000 nascidos vivos. Houve 2,5 vezes maior prevalência da anomalia em pacientes do sexo feminino, o que também é relatado na literatura ${ }^{(6,12,13)}$.

Em relação à ocorrência específica de mielomeningocele, a prevalência foi menor do que a encontrada por Ulsenheimer et al, que mostraram 1,8 casos a cada 1.000 nascidos vivos. Essa diferença possivelmente se deve ao fato de os autores acima citados terem estudado os casos em um serviço de referência para tais defeitos ${ }^{(14)}$.

Essas anomalias se mostraram associadas a características neonatais como peso ao nascer, duração da gestação e escore de Apgar. Quanto ao encontro de menor peso ao nascimento em portadores da malformação, os dados coincidem com outros estudos $^{(5-7)}$. Talvez tal associação ocorra pelo possível efeito da própria anomalia no crescimento fetal ou por um maior risco de presença de malformações no grupo de recém-nascidos de baixo peso. O peso médio foi menor nos neonatos portadores de defeitos no tubo neural em comparação aos sem defeito, assim como o risco de o paciente portador da anomalia nascer com baixo peso foi seis vezes maior.

Chamam atenção os valores do escore de Apgar que estimam a vitalidade neonatal. Os valores médios desse escore foram significantemente menores nos portadores de defeitos de tubo neural. Quando os pacientes foram categorizados em presença ou ausência de boa vitalidade ao nascer, o risco de o recém-nascido com a malformação apresentar baixa vitalidade foi 12 vezes maior, mostrando a influência da anomalia na vitalidade.

Outro achado importante foi a associação entre defeitos de tubo neural e prematuridade. A presença de nascimentos ocorridos antes de 37 semanas de gestação foi cerca de seis vezes maior dentre os portadores de defeitos no tubo neural. Mesmo considerando a possível existência de falhas no preenchimento do campo referente ao tempo de gestação na Declaração de Nascido Vivo, o risco de nascimento prematuro é elevado.

As variáveis relativas às mães não se mostraram associadas à presença da malformação. No caso da idade materna, o risco foi maior nas mães adolescentes e nas maiores de 37 anos, mas sem significância estatística.

As mães com menor escolaridade apresentaram chance mais de duas vezes maior de ter um neonato portador da malformação, embora sem significância estatística. A escolaridade materna é um indicador obstétrico ${ }^{(15)}$ e também está associada ao nível socioeconômico. Assim, mães com menor escolaridade podem ter dificuldade de acesso ao pré-natal, começando o acompanhamento da gestação mais tardiamente. Desta forma, a ingestão de ácido fólico só se inicia após o primeiro trimestre da gravidez, o que pode contribuir para o aumento de defeitos no tubo neural em conceptos de mães com baixa escolaridade. Da mesma forma, o risco de quase duas vezes para as mães com menor número de consultas no pré-natal pode ser explicado pelo possível início tardio do pré-natal e menor ingestão de ácido fólico.

A maior paridade também foi fator de risco para a anomalia, pois mães com dois ou mais filhos tinham risco maior de ter um filho com a anomalia em comparação às primíparas, contrapondo-se aos dados de Aguiar ${ }^{(5)}$, mas concordando com os de Castilla et $a l^{(16)}$. Costa et $a l^{(11)}$, no município do Rio de Janeiro, encontraram maior risco de defeitos no tubo neural em mães primíparas, mas a categorização das gestantes foi diferente da feita no presente estudo, dificultando a comparação.

Embora a literatura relate que a presença de óbito fetal anterior seja fator de risco para malformações ${ }^{(7)}$, tal variável não se associou ao nascimento de portadores da anomalia aqui analisada. Talvez a falta de informação nos 12 casos tenha comprometido o resultado.

A proporção de defeitos no fechamento do tubo neural no total de recém-nascidos com anomalias congênitas foi de $13,9 \%$, sendo esse valor intermediário aos relatados por Costa et al ${ }^{(11)}(9,9 \%)$ e Pacheco et al $l^{(6)}(31,5 \%)$.

Com o início precoce do pré-natal, a ingestão de ácido fólico ocorreria no começo da gestação, podendo exercer seu papel protetor com mais eficácia, embora deva ser enfatizada a importância da administração do suplemento vitamínico antes da concepção, a fim de que o efeito protetor dessa estratégia ocorra de forma plena. A maior atenção ao diagnóstico ultra-sonográfico do defeito no fechamento do tubo neural faria com que a gravidez fosse monitorada e possibilitaria um parto com maiores cuidados, dado o grande risco de o recémnascido portador da anomalia ter baixo peso, ser prematuro e apresentar um quadro de asfixia neonatal.

É importante lembrar que não foi objetivo deste estudo avaliar a qualidade de suplementação de ácido fólico às gestantes ou o cumprimento do programa do Ministério da Saúde ${ }^{(10)}$. Deve ser ressaltado o fato de que foram analisados dados secundários, presentes na Declaração de Nascido Vivo, o que pode ser uma limitação do estudo, pois este documento não contempla a ocorrência de defeitos múltiplos.

Apesar dessas limitações, os resultados indicam a necessidade de maior atenção no momento do parto em caso de diagnóstico de defeito no fechamento do tubo neural para que se previnam ou minimizem possíveis complicações. 


\section{Referências bibliográficas}

1. Northrup $\mathrm{H}$, Volcik KA. Spina bifida and other neural tube defects. Curr Probl Pediatr 2000;30:313-32.

2. Gelineau-van Waes J, Finnell RH. Genetics of neural tube defects. Semin Pediatr Neurol 2001;8:160-4. Semin Pediatr Neurol 2001;8:160-4

3. Frey L, Hauser WA. Epidemiology of neural tube defects. Epilepsia 2003;44 (Suppl 3):4-13

4. Melvin EC, George TM, Worley G, Franklin A, Mackey J, Viles K et al. Genetic studies in neural tube defects: NTD Collaborative Group. Pediatr Neurosurg 2000;32:1-9.

5. Aguiar MJ, Campos AS, Aguiar RA, Lana AM, Magalhães RL, Babeto LT. Defeitos de fechamento do tubo neural e fatores associados em recém-nascidos vivos e natimortos. J Pediatr (Rio J) 2003;79:129-34.

6. Pacheco SS, Souza Al, Vidal AS, Guerra GV, Batista-Filho M, Baptista EV et al. Neural tube defects prevalence in newborn infants in the women care center of the Instituto Materno Infantil Prof. Fernando Figueira, IMIP: 2000-2004. Rev Bras Saude Matern Infant 2006;6 (Suppl 1):S35-42.

7. Nascimento LF, Pinto CO, Proença FP, Gotlieb SL. Prevalência de anomalias congênitas em São José dos Campos, Brasil, 2001. Rev Paul Pediatr 2006;24:47-51.

8. Nazer-HJ, López-Camelo JS, Castilla EE. ECLAMC: Estudio de 30 años de vigilancia epidemiológica de defectos de tubo neural en Chile y en Latino América. Rev Med Chil 2001;129:531-9.
9. [No authors listed]. Folic acid for the prevention of neural tube defects. American Academy of Pediatrics. Committee on Genetics. Pediatrics 1999;104:325-7.

10. Brasil - Anvisa [homepage on the internet]. Legislação em vigilância sanitária. Resolução RDC $n^{0}$ 344, 13 dez 2002: aprova o regulamento técnico para a fortificação das farinhas de trigo e das farinhas de milho com ferro e ácido fólico [Cited 2008 Feb 11]. Available from: http://www.anvisa.gov.br/legis/ resol/2002/344_02rdc.htm

11. Costa CM, Gama SG, Leal MC. Congenital malformations in Rio de Janeiro, Brazil: prevalence and associated factors. Cad Saude Publica 2006;22:2423-31.

12. Stevenson RE, Allen HP, Pai GS, Best R, Seaver LH, Dean J et al. Decline in prevalence of neural tube defects in a high-risk region of the United States. Pediatrics 2000;106:677-83.

13. Hunter GW. Brain and Spinal Cord. In: Stevenson ER, Hall JG, Goodman RM, editors. Human malformations and related anomalies. New York: Oxford University Press; 1993. p. 109-31.

14. Haidar FH, Oliveira UF, Nascimento LF. Escolaridade Materna: correlação com os indicadores obstétricos. Cad Saude Publica 2001;17:1025-9.

15. Ulsenheimer MM, Antoniuk SA, Santos LH, Ceccatto MP, Silveira AE, Ruiz AP et al. Mielomeningocele: experiência de um hospital universitário brasileiro. Arq Neuro-Psiquiatr 2004;62:963-8.

16. Castilla EE, Lopez-Camelo JS, Paz JE, Orioli IM. Prevervação primária dos defeitos congênitos. Rio de Janeiro: Editora Fiocruz; 1996. 